\title{
Characterizing microclimate in urban malaria transmission settings: a case study from Chennai, India
}

Lauren J Cator ${ }^{1,4}$, Shalu Thomas ${ }^{2}$, Krijn P Paaijmans', Sangamithra Ravishankaran², Johnson A Justin², Manu T Mathai ${ }^{3}$, Andrew F Read ${ }^{1,4}$, Matthew B Thomas ${ }^{1,4}$ and Alex Eapen ${ }^{2 *}$

\begin{abstract}
Background: Environmental temperature is an important driver of malaria transmission dynamics. Both the parasite and vector are sensitive to mean ambient temperatures and daily temperature variation. To understand transmission ecology, therefore, it is important to determine the range of microclimatic temperatures experienced by malaria vectors in the field.

Methods: A pilot study was conducted in the Indian city of Chennai to determine the temperature variation in urban microclimates and characterize the thermal ecology of the local transmission setting. Temperatures were measured in a range of probable indoor and outdoor resting habitats of Anopheles stephensi in two urban slum malaria sites. Mean temperatures and daily temperature fluctuations in local transmission sites were compared with standard temperature measures from the local weather station. The biological implications of the different temperatures were explored using temperature-dependent parasite development models to provide estimates of the extrinsic incubation period (EIP) of Plasmodium vivax and Plasmodium falciparum.

Results: Mean daily temperatures within the urban transmission sites were generally warmer than those recorded at the local weather station. The main reason was that night-time temperatures were higher (and hence diurnal temperature ranges smaller) in the urban settings. Mean temperatures and temperature variation also differed between specific resting sites within the transmission environments. Most differences were of the order of $1-3^{\circ} \mathrm{C}$ but were sufficient to lead to important variation in predicted EIPs and hence, variation in estimates of transmission intensity.

Conclusions: Standard estimates of environmental temperature derived from local weather stations do not necessarily provide realistic measures of temperatures within actual transmission environments. Even the small differences in mean temperatures or diurnal temperature ranges reported in this study can lead to large variations in key mosquito and/or parasite life history traits that determine transmission intensity. Greater effort should be directed at quantifying adult mosquito resting behaviour and determining the temperatures actually experienced by mosquitoes and parasites in local transmission environments. In the absence of such highly resolved data, the approach used in the current study provides a framework for improved thermal characterization of transmission settings.
\end{abstract}

Keywords: Temperature, Extrinsic incubation period, Anopheles stephensi, Urban malaria

\footnotetext{
* Correspondence: alexeapen@yahoo.com

${ }^{2}$ National Institute of Malaria Research (ICMR), IDVC Field Unit, NIE Campus, 2nd Main Road, TNHB, Ayapakkam, Chennai 600 077, India

Full list of author information is available at the end of the article
} 


\section{Background}

Temperature is one of the key environmental factors influencing the dynamics and distribution of malaria. Various studies show that mosquito population dynamics [1-3], frequency of blood feeding [4], parasite fitness in the vector [5], mosquito immune processes [6] and the extrinsic incubation period (EIP) of the parasite within the mosquito $[7,8]$ are all affected by temperature.

Currently, the majority of studies considering the effect of temperature on mosquito bionomics and malaria risk use temperatures recorded from standard outdoor weather stations [9-16]. However, temperatures from weather stations, which are often separated from transmission sites, do not necessarily represent the temperatures experienced by vectors in local transmission settings in the field [17]. Temperature can vary greatly between indoor and outdoor environments and also be influenced strongly by local features such as house design, house materials, and vegetation cover [3,17-20]. Such differences can translate to marked variation in mosquito life history and estimates of malaria transmission [17].

Further, most studies characterize the environmental conditions using measures of temperature such as mean monthly temperatures, yet recent theoretical [21] and empirical [22] work has demonstrated that daily fluctuations in temperature also affect mosquito and parasite traits that determine transmission processes. In order to understand the influence of temperature on transmission, temperature needs to be measured within the actual environment inhabited by mosquito vectors and at a scale relevant to mosquito and parasite biology. The current paper presents the results of a pilot study, providing a basic methodological approach for addressing these issues.

The study was conducted in the city of Chennai in Tamil Nadu, southeastern India, where the predominant malaria vector is Anopheles stephensi. This species is an important vector of malaria throughout the Indian subcontinent and has been reported to rest both indoors [23-26] and outdoors [23]. Data from mechanical aspiration and pyrethrum spray catches, in which a home is sealed and pyrethrum fog is applied to capture all knocked-down mosquitoes on white sheets [27], in India have documented this species resting in a variety of indoor structures [23-25], as well as in semi-outdoor habitats such as cattle sheds [25]. The majority of $A n$. stephensi females were found to leave indoor resting habitats early in the evening (18.30 hrs.), feed primarily outdoors, and then return to indoor resting habitats in the middle of the night (23.30 hrs.) [23]. However, there seems to be plasticity in peak biting times with one study reporting two peaks of activity [28].

The biting and resting behaviour of An. stephensi within urban slum transmission sites in Chennai is not well characterized, although adults have been sampled periodically from a range of indoor and outdoor structures. In the absence of more explicit knowledge, the current study describes methods for recording temperature in a range of potential indoor and outdoor resting locations in order to capture the broad 'thermal envelope' available within the local transmission settings. These diverse temperature data were then used to drive thermodynamic models of parasite development to examine the expected variation in the Extrinsic Incubation Period (EIP) of malaria parasites across the transmission environment. The logic is that in the absence of precise knowledge of mosquito resting behaviour (and given that mosquitoes almost certainly distribute across a range of microhabitats during their adult life) the transmission environment is better characterized as an ensemble mean of available habitats with an upper and lower bound derived from the local conditions, rather than a single value based on mean temperature from a remote weather station.

\section{Methods}

\section{Field sites and sampling rationale}

The study was conducted in the catchment areas of two malaria clinics. Both George Town $\left(13.0939^{\circ} \mathrm{N}, 80.2839^{\circ} \mathrm{E}\right)$ and Besant Nagar $\left(13.0002^{\circ} \mathrm{N}, 80.2668^{\circ} \mathrm{E}\right)$ clinics consistently report positive cases of both Plasmodium vivax and Plasmodium falciparum malaria that are transmitted by An. stephensi. Malaria cases reported at Besant Nagar clinic from 2006 to 2011 were higher compared to that of George Town. In Besant Nagar, the Slide Positivity Rate (SPR) varied from 22.6 to 42.6 , with Pf\% of 2.6 to $13.7 \%$. The corresponding values reported at George Town ranged from 9.59 to 15.8 and 0.32 to $0.9 \%$, respectively (Source: Malaria clinic data, Corporation of Chennai, India).

The current investigation was designed as a preliminary study to develop appropriate protocols and methods for a proposed year-round longitudinal survey combining environmental monitoring along with adult and larval sampling within these transmission environments. This pilot study was conducted from late January to mid April of 2012, which represents a relatively low transmission period after the end of the northeast monsoon season of October to December 2011.

\section{Placement and download of temperature data loggers}

Forty-two data loggers were placed across the transmission sites. The majority of the loggers were small 'USBmode' loggers set to record temperature every 30 minutes (USB-Lite, Dwyer Instruments, Michigan City, IN, USA). These loggers were selected because they were relatively inexpensive and there was no prior knowledge of how many loggers might be lost during the study. The USB loggers were supplemented with a few more expensive Hobo loggers (HOBO U12-011, Onset, Cape Cod, MA, USA) to compare reliability. 
Temperatures were recorded from both indoor and outdoor sites; different structure types and homes constructed using a range of materials (Table 1). Indoor structures included positions within tile-roofed, asbestosroofed, concrete-roofed, and thatched-roofed homes. Outdoor loggers were placed in vegetation, wells, and overhead tanks. Three loggers were placed in 'other outdoor' locations; one in a crevice of an outdoor brick wall, one under the thatched roof of an outdoor porch and another hanging in an outdoor terrace. Within the habitats, loggers were placed in areas typically utilized by mosquitoes (e.g. in dark corners, behind furniture, within shaded vegetation, hanging inside a well etc.) rather than exposed microenvironments where it would be unlikely to find a resting adult. The position of each logger was recorded using a GPS and details of the location and structure/habitat recorded. Loggers were downloaded every two weeks. Loggers recorded from early January to April 2012 with a peak coverage period of February 6, 2012 through April
16, 2012. Due to logger malfunction or loss, the sample size varies slightly for each month (Table 1). Additionally, hourly weather station data for the city of Chennai (actually at the airport) were downloaded from the National Oceanic and Atmospheric Administration (NOAA), National Climatic Data Center [29]. The Besant Nagar clinic is located approximately $15 \mathrm{~km}$ west of the local weather station at Meenambakam Airport. The George Town field site is approximately $20 \mathrm{~km}$ northwest of this weather station

\section{Data analysis}

For each logger, on each day the minimum, maximum, mean and temperature range (DTR, the difference between daily minimum and maximum) were calculated. Additionally, the hourly temperature recorded by each logger was averaged each day to give a daily mean temperature for each logger. The average temperatures reported for loggers located in different field sites (NOAA,

Table 1 Mean, minimum and maximum temperature, and mean DTR

\begin{tabular}{|c|c|c|c|c|c|c|c|}
\hline Month & Habitat/Area & Structure type & $\mathrm{n}$ & $\begin{array}{l}\text { Mean temperature } \\
\qquad\left({ }^{\circ} \mathrm{C} \pm \mathrm{SE}\right)\end{array}$ & $\begin{array}{l}\text { Min } \\
\left({ }^{\circ} \mathrm{C}\right) \\
\end{array}$ & $\begin{array}{l}\text { Max } \\
\left({ }^{\circ} \mathrm{C}\right) \\
\end{array}$ & $\begin{array}{c}\text { Mean DTR } \\
\left({ }^{\circ} \mathrm{C} \pm \mathrm{SE}\right) \\
\end{array}$ \\
\hline \multirow[t]{9}{*}{ February } & Indoor & Asbestos Roof & 5 & $30.20 \pm 0.02$ & 25 & 36 & $4.39 \pm 0.15$ \\
\hline & & Concrete Roof & 9 & $29.60 \pm .02$ & 24 & 37 & $2.15 \pm 0.18$ \\
\hline & & Thatch Roof & 3 & $28.80 \pm 0.08$ & 24 & 36 & $5.78 \pm 0.22$ \\
\hline & & Tile Roof & 8 & $29.92 \pm 0.02$ & 25 & 35 & $3.40 \pm 0.03$ \\
\hline & Outdoor & Vegetation & 5 & $27.41 \pm 0.03$ & 21 & 35 & $6.21 \pm 0.25$ \\
\hline & & Well & 3 & $27.97 \pm 0.03$ & 22 & 36 & $3.27 \pm 0.45$ \\
\hline & & Overhead Tank & 1 & $32.88 \pm 0.15$ & 26 & 45 & $14.88 \pm 0.45$ \\
\hline & & Other Outdoor & 3 & $29.37 \pm 0.04$ & 22 & 36 & $6.10 \pm 0.28$ \\
\hline & & NOAA & 1 & $26.26 \pm 0.10$ & 20 & 35 & $9.67 \pm 0.36$ \\
\hline \multirow[t]{9}{*}{ March } & Indoor & Asbestos Roof & 6 & $31.89 \pm 0.02$ & 28 & 39 & $3.99 \pm 0.10$ \\
\hline & & Concrete Roof & 7 & $30.84 \pm 0.01$ & 27 & 36 & $1.37 \pm 0.06$ \\
\hline & & Thatch Roof & 3 & $30.19 \pm 0.04$ & 26 & 38 & $5.13 \pm 0.21$ \\
\hline & & Tile Roof & 8 & $31.50 \pm 0.02$ & 25 & 40 & $3.83 \pm 0.13$ \\
\hline & Outdoor & Vegetation & 5 & $29.79 \pm 0.02$ & 25 & 38 & $6.03 \pm 0.20$ \\
\hline & & Well & 3 & $29.31 \pm 0.03$ & 25 & 40 & $3.64 \pm 0.39$ \\
\hline & & Overhead Tank & 1 & $33.73 \pm 0.10$ & 27 & 43 & $12.03 \pm 0.25$ \\
\hline & & Other Outdoor & 3 & $31.36 \pm 0.04$ & 25 & 37 & $7.18 \pm 0.33$ \\
\hline & & NOAA & 1 & $28.94 \pm 0.08$ & 22 & 36 & $9.07 \pm 0.30$ \\
\hline \multirow[t]{9}{*}{ April } & Indoor & Asbestos Roof & 6 & $33.01 \pm 0.03$ & 29 & 39 & $4.41 \pm 0.14$ \\
\hline & & Concrete Roof & 7 & $31.57 \pm 0.01$ & 30 & 35 & $0.94 \pm 0.07$ \\
\hline & & Thatch Roof & 2 & $30.85 \pm 0.05$ & 27 & 36 & $3.97 \pm 0.21$ \\
\hline & & Tile Roof & 8 & $32.36 \pm 0.02$ & 28 & 38 & $3.70 \pm 0.16$ \\
\hline & Outdoor & Vegetation & 4 & $30.69 \pm 0.04$ & 19 & 35 & $5.19 \pm 0.25$ \\
\hline & & Well & 3 & $30.25 \pm 0.03$ & 27 & 37 & $4.00 \pm 0.46$ \\
\hline & & Overhead Tank & 1 & $34.76 \pm 0.15$ & 29 & 43 & $11.38 \pm 0.59$ \\
\hline & & Other Outdoor & 3 & $32.35 \pm 0.05$ & 27 & 40 & $6.38 \pm 0.38$ \\
\hline & & NOAA & 1 & $30.22 \pm 0.12$ & 24 & 37 & $9.10 \pm 0.35$ \\
\hline
\end{tabular}


Besant Nagar, and George Town) were compared using 95\% confidence intervals. Differences between temperatures reported by data loggers located in the same structure type, but in different field sites, were determined using a paired t-test [30]. All statistical analyses were run using SPSS (Version 20.0. IBM Corporation, Armonk, NY). Mean monthly temperatures (mean of daily temperatures in a month) and mean DTR (mean of daily temperatures recorded) were also calculated for each logger type for use in modelling parasite development.

\section{Thermodynamic models}

The effects of temperature (T) on the EIP of the parasite was examined using a published non-linear thermodynamic model for $P$. falciparum [21]. This model describes parasite development rate (PDR, the reciprocal of the EIP) and was derived by fitting a standard temperature-development function [31] to a range of published empirical and modelling data on parasite development:

$$
P D R_{\text {falciparum }}(T)=0.000112 T(T-15.384) \sqrt{(35-T)}
$$

A similar curve was generated for P. vivax development using a suite of empirical data [32-37] combined with estimates of development from the standard degree-day model for P. vivax [38] over the linear range of the Brière function (Figure 1) to give:

$$
\begin{aligned}
P_{D} R_{\text {vivax }}(T)= & 0.000126 T(T-14.244) \\
& \sqrt{(34.4-T)} \quad\left(R^{2}=0.897\right)
\end{aligned}
$$

One empirical data point from a study in South America was omitted from the analyses, as it suggested a critical

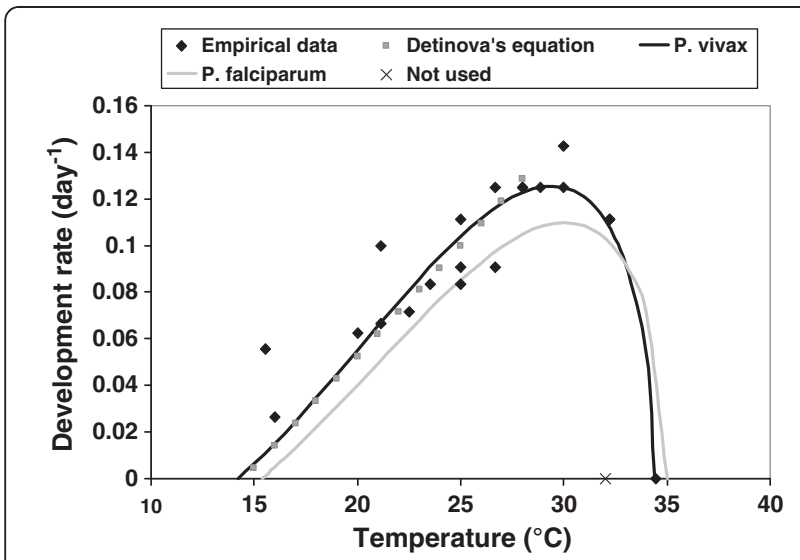

Figure 1 Relationship between temperature and the development rate of $P$. vivax. The function as proposed by Brière et al. [30] is fitted to a set of empirical data (see Methods for references) and the well-established Detinova equation [37] over a defined temperature range (black line). The previously published thermal performance curve for $P$. falciparum is plotted in grey. The optimal temperature for $P$. falciparum development in this curve is $30.1^{\circ} \mathrm{C}$ and $29.4^{\circ} \mathrm{C}$ for P. vivax. maximum temperature $\left(\mathrm{CT}_{\max }\right)$ for $P$. vivax of $32^{\circ} \mathrm{C}$, yet two other studies in Indian vectors indicate parasite development above this temperature [33,34].

Mean hourly temperature and mean monthly temperature data for each data logger were used to calculate the development rates of $P$. falciparum and $P$. vivax using the thermodynamic models presented above, and subsequently converted to provide estimates of EIP. Average EIPs for each structure type were compared across each month, based on either the mean monthly temperatures (the typical way of characterizing the environment) or the mean hourly temperatures, which captures any additional effect of temperature variation that occurs through the day. The temperature development models include an upper lethal limit, set at $5^{\circ} \mathrm{C}$ above the $\mathrm{CT}_{\max }$ [39]. If temperatures exceed these limits the parasite is assumed to die and no estimates of EIP are possible. Curve fitting was done in SPSS (IBM SPSS v20), with the analyses and figures generated in $\mathrm{R}$ [40].

\section{Results}

Comparison between weather station data and temperatures within the urban transmission sites

Environmental temperature increased over the two and a half months (February, March, first half of April) monitoring period (Figure 2A). Average daily temperatures recorded from data loggers within the local transmission sites were significantly warmer than those recorded by the NOAA weather station (Figure 2A). There were no differences between the temperatures recorded by loggers located in the same structure types between the field sites (paired $t$-test, $t=0.92$, d.f. $=6, t=0.39$ ). The lower average daily temperatures reported by the weather station were due to the fact that this location experienced cooler temperatures during the night. This effect also resulted in larger average DTRs at this location compared with the urban transmission (Figure 2B).

Diversity of thermal environments within transmission sites Indoor environments were warmer than outdoor environments (Indoor, 95\% Confidence Interval, 30.86-30.89; Outdoor, 95\% Confidence Interval, 29.99-30.06) and had smaller DTRs (Indoor, 95\% Confidence Interval, 3.203.41; Outdoor, 95\% Confidence Interval, 5.92-6.43). As mean temperatures increased over the sample period, DTRs generally decreased (Figure 3). The one exception to this pattern was an overhead water storage tank located on the roof of a 5-storey apartment building that had the warmest temperatures and the largest DTRs. Among the other environments, homes with tile and asbestos roofs were the warmest, while outdoor vegetation and wells were the coolest (Table 1). Loggers placed in 'other outdoor' locations had the largest DTRs, and the 

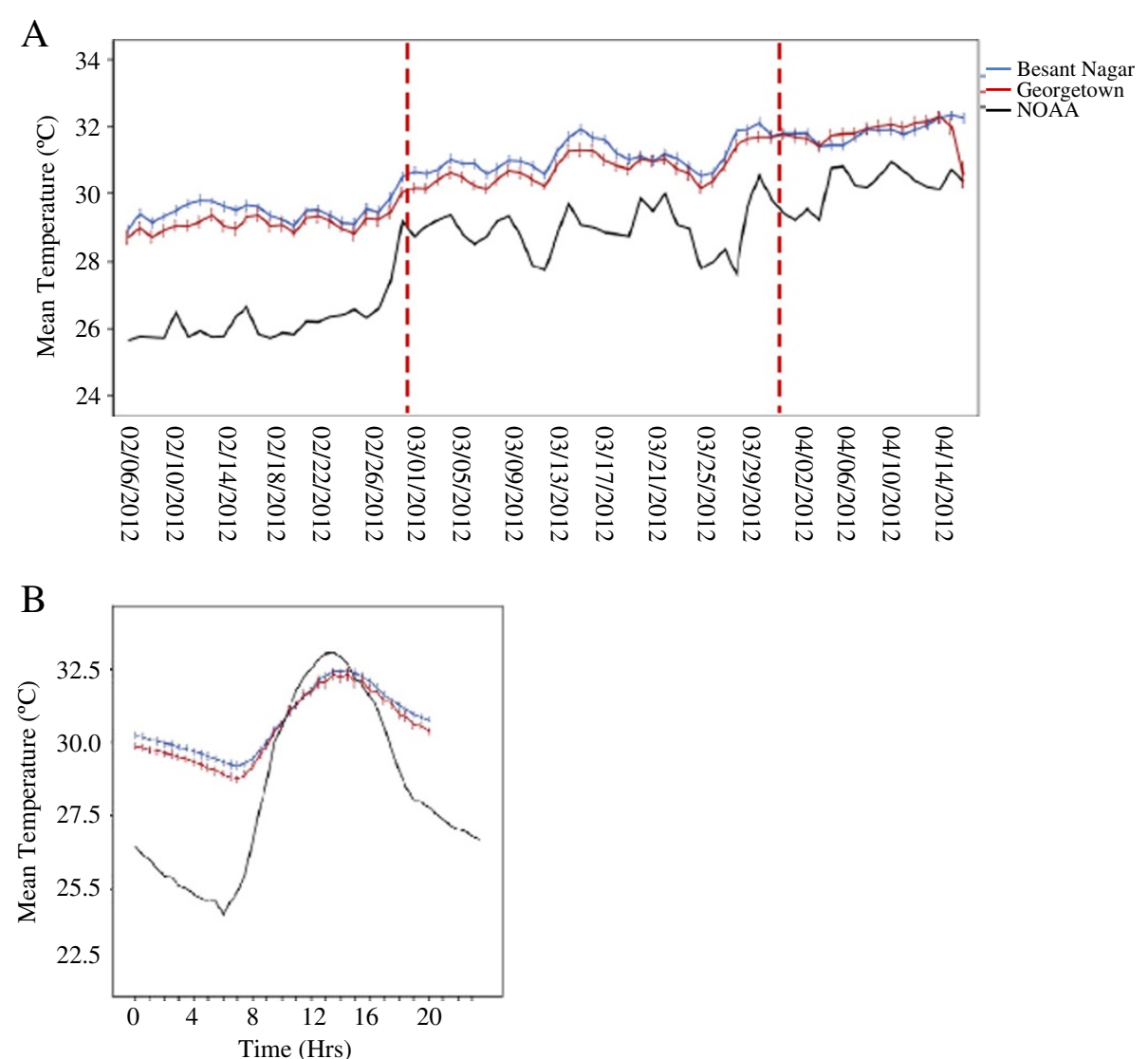

Figure 2 Temperature reported by local weather station and within transmission sites. A. Average daily temperature over sampling period in all sites. Red lines indicate the month breaks in the data. B. Average hourly temperature profile for loggers located at transmission sites and the nearest NOAA weather station. Within transmission sites there was less cooling during the night and this resulted in higher average temperatures. Bars represent approximate $95 \%$ confidence intervals ( \pm 2 standard errors) of the mean calculated across the hourly temperature for loggers at each site.

smallest DTRs were recorded from the inside of houses with concrete roofs.

\section{Extrinsic incubation periods}

With the exception of the overhead tank (Figure 4), the predicted EIPs in all indoor and outdoor structures were comparable in February (P. vivax 8-9 days; P. falciparum 9-10 days). However, all had predicted EIPs 1-2 days shorter than those predicted using the weather station data (Figure 5). As conditions warmed, predicted EIPs deviated slightly between microenvironments. The more stable conditions $(<1$ day difference in predicted EIP, comparing February with April) were indoors in concrete-roofed and thatch-roofed houses, and outdoors in vegetation and wells. The houses with asbestos and tile roofs (indoor) and other outdoor habitats showed larger increases in predicted EIP over time. The shortest EIPs were predicted to be in warm stable habitats such as concrete and thatch-roofed houses and wells (Figure 4). Across the different environments, predicted EIPs ranged from 2 days shorter to 3 days longer than the EIPs based on the weather station temperatures.
Temperatures in the overhead tank were well beyond the optimum temperature for $P$. falciparum and $P$. vivax development (Figure 4). Increases in temperature over the 3 months in this structure led to substantial changes in predicted EIP for P. falciparum and blocked development of $P$. vivax as mean temperatures exceeded the $\mathrm{CT}_{\max }$ (Figure 4). For most resting habitats, incorporating the effects of daily temperature fluctuations had small effects on predicted EIP, acting generally to reduce variation between microenvironments. Effects of fluctuation were slightly more marked for the predicted EIPs calculated using weather station data as the daily temperature variation reported from this location was roughly double that of most other habitat types. For these data, temperature variation acted to prolong predicted EIP in the warmest period relative to mean temperatures alone (Figure 4).

As a method to consolidate these diverse data, the estimates of the EIPs from the different microhabitat types were averaged to provide an 'ensemble mean' for the local transmission settings and were coupled with the 

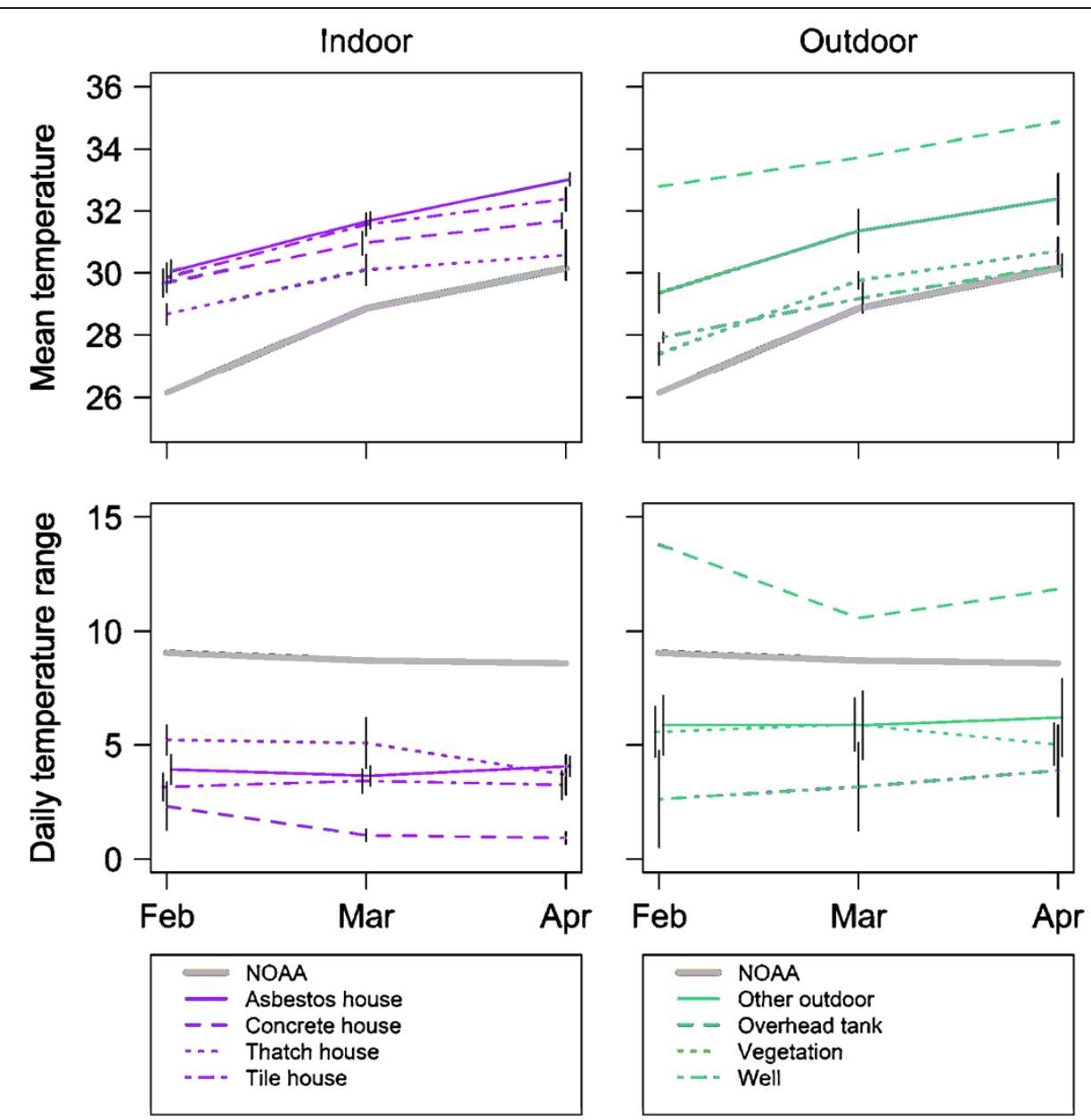

Figure 3 Average temperature and DTR for different structure types (divided in indoor and outdoor structures) over a 3 month time period. Bars represent standard errors; there are no error bars in cases in which measurements were from a single logger (NOAA and overhead tank).

upper and lower bounds of possible EIPs based on the existing environmental extremes (Figure 5). This method of presenting an ensemble mean together with an estimate of the range follows the approach commonly used to synthesize outputs from multiple Global Climate Models. In most instances, the EIP calculated from the ensemble mean differed from the estimates of EIP based on weather station data (differences in absolute value and also trends across time). More striking is the extent of the environmental envelope representing potential EIPs within the transmission setting. These extremes of potential EIP sometimes deviate from the weather station estimates by $>20$ days (note that in some cases predicted range of potential EIP declines as certain microenvironments become too hot to support successful parasite development).

\section{Discussion}

The primary aim of the current study was to determine methods for sampling temperatures within transmission environments and to propose these as a framework for better understanding local transmission ecology. The empirical data presented serve as a pilot study for a more extensive longitudinal monitoring programme and so are relatively limited in scope (i.e. they are not themselves intended to provide an exhaustive evaluation of the transmission environment in the urban slums in Chennai). Nonetheless, the study illustrates the benefits of examining the variation in temperature between different potential mosquito resting habitats and potential implications for malaria transmission.

Most studies that consider the role of temperature in malaria transmission use temperatures reported by local weather stations. The current study revealed that the temperatures within the local transmission sites were warmer and more varied than those recorded by the weather station at the airport. The temperature loggers were located in a densely packed urban environment that showed less cooling at night (Figure 2B). Such 'urban heat islands' have been reported elsewhere in the 


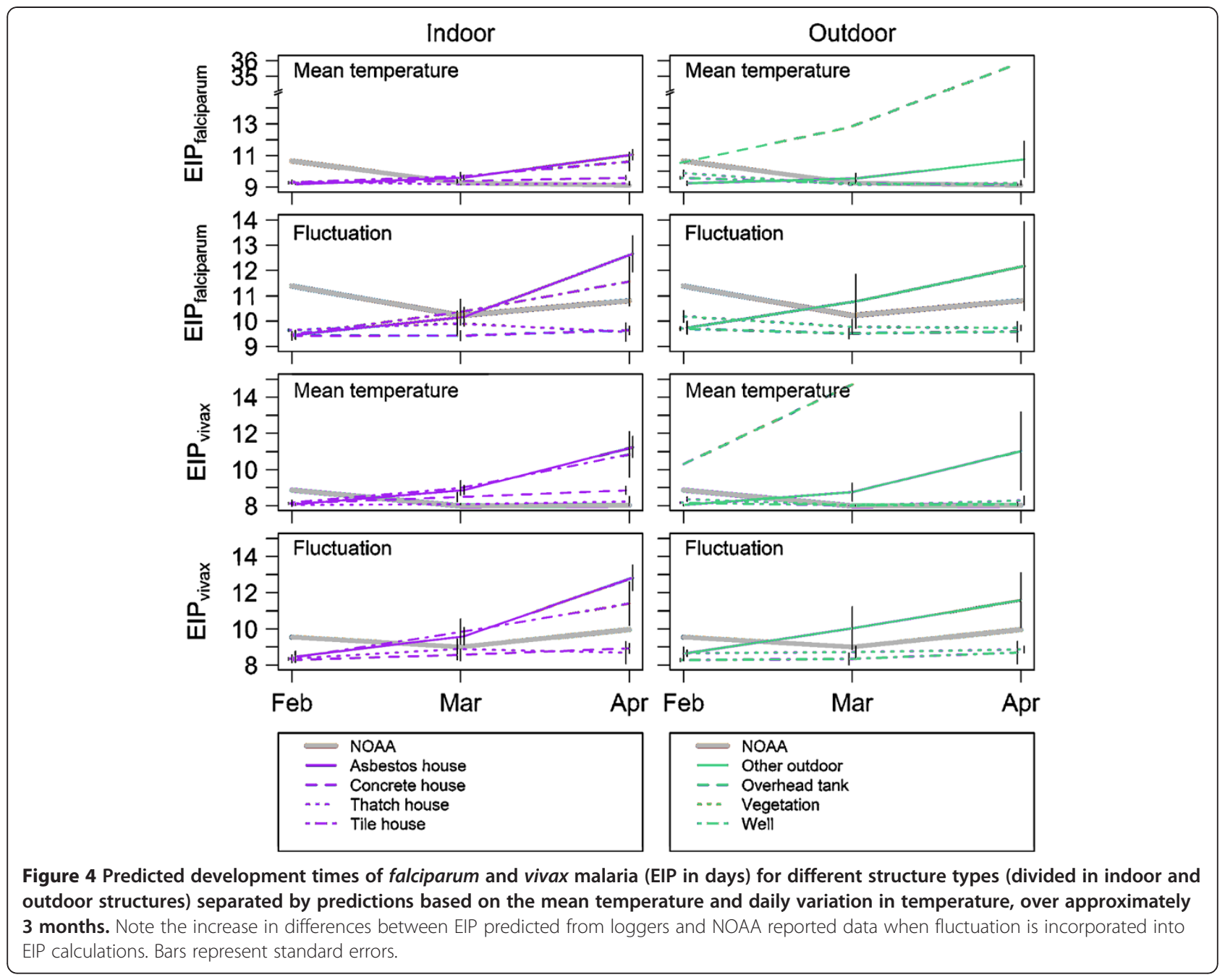

literature $[41,42]$ and tend to experience higher average daily temperatures than surrounding areas via effects on night-time temperatures.

Within the transmission sites, indoor temperatures remained warmer and were more stable than those recorded for outdoor environments. This type of thermal buffering has been reported in other studies, both in general terms [43] and with specific reference to malaria transmission $[3,44]$. With the exception of the overhead tank, which proved to be something of an extreme environment, differences in mean temperatures between microhabitats were relatively small. Even so, variation between environments led to differences in the predicted EIPs of 1-4 days for both P. falciparum and P. vivax. Adding the effects of daily temperature variation had relatively little effect because, in contrast to other parts of the world [17], the DTRs themselves were relatively small and consistent across habitats.

Anopheles stephensi exhibits both endophilic (indoor resting) and exophilic (outdoor resting) behaviour. The current study took a broad snap shot of temperatures available for adult mosquitoes, but it is possible that $A n$. stephensi only utilizes a subset of these environments. Further, temperatures will likely vary within structure types (i.e. at different positions within a single house type) and so the temperatures experienced by mosquitoes could depend on the subtleties of the precise resting position. There is some indication that adult anopheline mosquitoes can avoid the warmest locations [45], but there is little evidence for precise behavioural thermoregulation [46]. Additionally, it has been shown that expression of mosquito heat-shock proteins increase in response to thermal stress [47] and these proteins have been shown to interact with Plasmodium development [48]. The extent to which these interactions would affect EIP in these environments is unclear, but should be considered in future studies. Further study on the activity patterns of An. stephensi in this urban environment and resting preferences within and between structure types is clearly required. For the current study there was no 


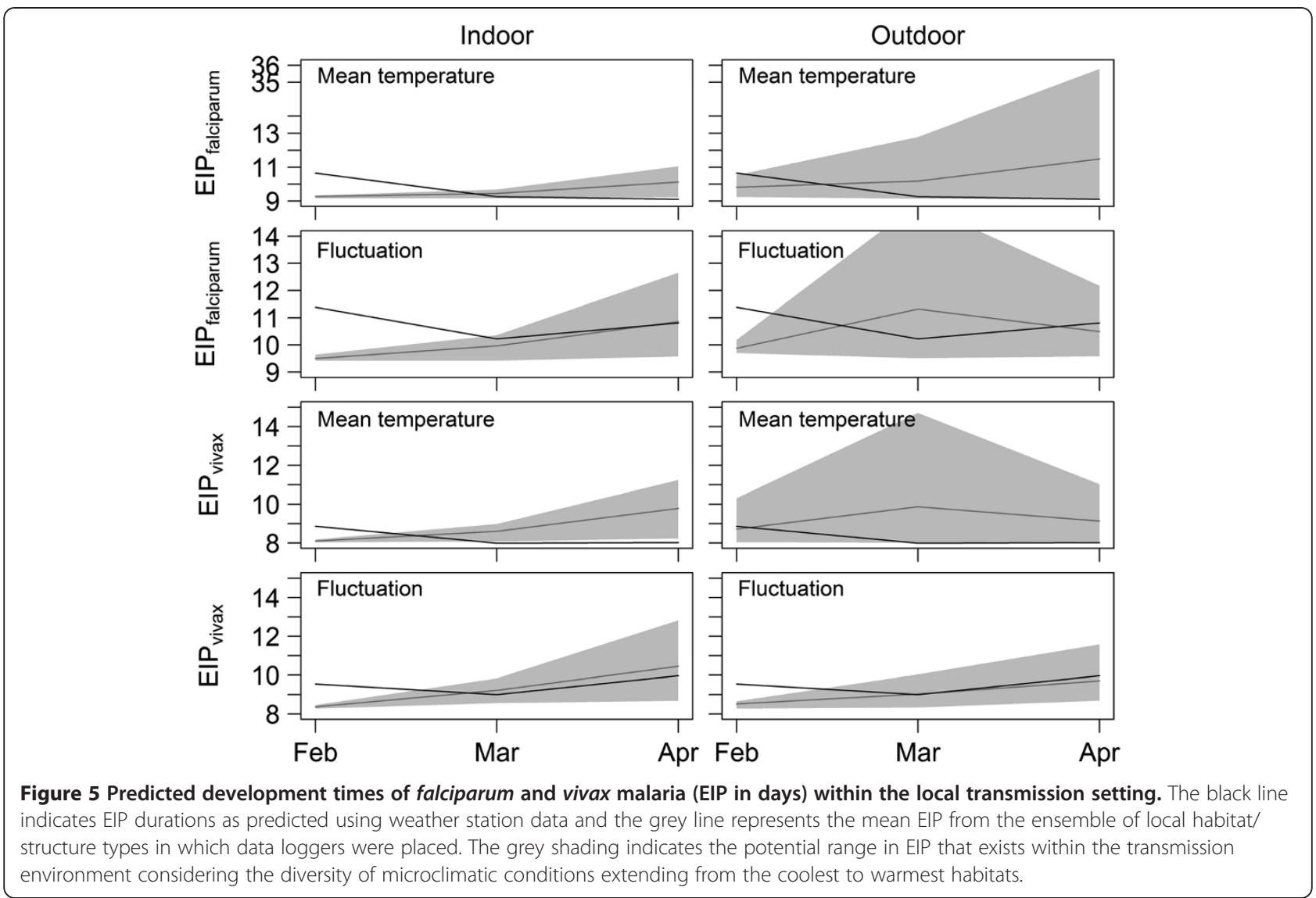

systematic mosquito sampling, although case data from the malaria clinics indicated there was active malaria transmission in these areas during the study period and immature forms of An. stephensi were found in the field sites, indicating that An. stephensi was utilizing some portion of the environments sampled. Precise behavioural data coupled with appropriate high resolution environmental data would refine understanding of the temperatures mosquitoes experience during parasite development. However, very few studies characterize mosquito resting behaviour in detail or couple entomological measures with site-specific estimates of microclimate.

Local meteorological station data provide a single measure of temperature and hence, generate a single estimate of temperature-dependent traits such as EIP. In contrast, the multiple data loggers placed within the transmission environment provide a measure of the temperature envelope in which mosquitoes live. In the current study, the EIPs based on the weather station data did not consistently align with the EIPs based on the ensemble mean temperatures derived from the different microhabitat types and clearly failed to capture the enormous amount of potential variation that exists within the transmission setting. The use of a distribution of temperatures does not make predictions of life history traits more 'precise', but it does make them more accurate in the sense that they represent the actual environment where mosquitoes rest and transmission occurs. If a single measure is required, the use of an 'ensemble mean' based on the average temperatures recorded across the local microhabitats should provide a more robust characterization of the transmission environment than the remote meteorological station data.

This study emphasizes the complexity of the thermal environment and its equally complex interaction with parasite development. Understanding transmission dynamics requires some consideration of this complexity. Even small absolute changes in EIP of 1-3 days can have marked impacts on transmission risk. The average daily probability of survival for An. stephensi has been estimated as 0.810 [49]. With an EIP of 10 days this means that approximately $12 \%$ of adult mosquitoes would live long enough to be able to transmit malaria. Increasing EIP to 12 days reduces this percentage to $8.5 \%$, while reducing EIP to 8 days increases the value to about $18.5 \%$. All else being equal, these changes would alter transmission intensity by approximately $-45 \%$ to $+50 \%$ [17] .

Gaining knowledge of the temperatures experienced by mosquito vectors in the field is an important step towards a better understanding of temperature as a driver 
of malaria transmission. Data on the natural thermal environments of vectors are also valuable for contextualizing laboratory work on mosquito-parasite interactions. The majority of laboratory studies on vector competence and host-parasite interactions are conducted at a constant temperature of around $26^{\circ} \mathrm{C}$. While only a limited study, the data from the urban transmission sites in Chennai suggest mosquitoes rarely encounter such temperatures and are subject to both higher mean temperatures and daily temperature fluctuations.

\section{Competing interests}

The authors declare that they have no competing interests.

\section{Authors' contributions}

MBT and AE designed the experiment with inputs from MTM and AFR. LC participated in study design and wrote the manuscript with assistance from MBT and AE. ST, SR and JAJ executed data logger placements and systematically downloaded the data in the field. KPP, ST and SR contributed to temperature data analysis. KPP applied models to temperature data collected. All authors read and approved the final manuscript.

\section{Acknowledgements}

We thank Director, NIMR (ICMR) for providing the necessary facilities and support. We gratefully acknowledge the staff of the NIMR Chennai field unit besides, J. Carlton, L. V. Ramanathapuram, P. Sutton, S. Wassmer for logistical support, and the communities of Besant Nagar, George Town for allowing us to record temperatures in their premises. This study was supported by the NIH-NIAID ICEMR award (\#U19AI089676) and the manuscript has been approved by NIMR publication screening committee (No. 029/2012). The content is solely the responsibility of the authors and does not necessarily represent the official views of the National Institutes of Health.

\section{Author details}

'Department of Entomology, Center for Infectious Disease Dynamics and Department of Entomology, Merkle Lab, The Pennsylvania State University, University Park, PA 16802, USA. ${ }^{2}$ National Institute of Malaria Research (ICMR), IDVC Field Unit, NIE Campus, 2nd Main Road, TNHB, Ayapakkam, Chennai 600 077, India. ${ }^{3}$ Department of Zoology, Madras Christian College, Tambaram, Chennai 600 059, India. ${ }^{4}$ Center for Infectious Disease Dynamics, The Pennsylvania State University, University Park, 16802, PA, USA.

Received: 24 December 2012 Accepted: 26 February 2013 Published: 2 March 2013

\section{References}

1. Parham PE, Michael E: Modeling the effects of weather and climate change on malaria transmission. Environ Health Perspect 2010, 118:620-626.

2. Paaijmans KP, Imbahale SS, Thomas MB, Takken W: Relevant microclimate for determining the development rate of malaria mosquitoes and possible implications of climate change. Malar J 2010, 9:196.

3. Afrane YA, Zhou G, Lawson BW, Githeko AK, Yan G: Effects of microclimatic changes caused by deforestation on the survivorship and reproductive fitness of Anopheles gambiae in western Kenya highlands. Am J Trop Med Hyg 2006, 74:772-778.

4. Rua GL, Quinones ML, V'elez ID, Zuluaga JS, Rojas W, Poveda G, Ruiz D: Laboratory estimation of the effects of increasing temperatures on the duration of gonotrophic cycle of Anopheles albimanus (Diptera: Culicidae). Mem Instit Oswaldo Cruz 2005, 100:515-520.

5. Paaijmans KP, Blanford S, Chan BHK, Thomas MB: Warmer temperatures reduce the vectorial capacity of malaria mosquitoes. Biol Lett 2011, 8:465-468.

6. Murdock CC, Paaijmans KP, Bell AS, King JG, Hillyer JF, Read AF, Thomas MB: Complex effects of temperature on mosquito immune function. Proc $R$ SOC B 2012, 279:3357-3366.

7. MacDonald G: The epidemiology and control of malaria. London: Oxford University Press; 1957.

8. Boyd M: Malariology: A comprehensive survey of all aspects of this group of diseases from a global standpoint. Philadelphia: W.B. Saunders Company; 1949.
9. Killeen GF, McKenzie FE, Foy BD, Schieffelin C, Billingsley PF, Beier JC: A simplified model for predicting malaria entomologic inoculation rates based on entomologic and parasitologic parameters relevant to control. Am J Trop Med Hyg 2000, 62:535-544.

10. Hay SI, Snow RW: The Malaria Atlas Project: developing global maps of malaria risk. PLoS Med 2006, 3:e473.

11. Guerra CA, Gikandi PW, Tatem AJ, Noor AM, Smith DL, Hay SI, Snow RW: The Limits and intensity of Plasmodium falciparum transmission: implications for malaria control and elimination worldwide. PLoS Med 2008, 5:e38.

12. Rogers DJ, Randolph SE: Climate change and vector-borne diseases. Adv Parasitol 2006, 62:345-381.

13. Craig MH, Snow RW, le Sueur D: A climate-based distribution model of malaria transmission in sub-Saharan Africa. Parasitol Today 1999, 15:105-111.

14. Martens P, Kovats RS, Nijhof S, De Vries P, Livermore MTJ, Bradley DJ, Cox J, McMichael AJ: Climate change and future populations at risk of malaria. Global Environ Change 1999, 9:S89-S107.

15. Rogers DJ, Randolph SE: The global spread of malaria in a future, warmer world. Science 2000, 289:1763-1766

16. Ebi KL, Hartman J, Chan N, McConnell J, Schlesinger M, Weyant J: Climate suitability for stable malaria transmission in Zimbabwe under different climate change scenarios. Clim Chang 2005, 73:375-393.

17. Paaijmans KP, Thomas MB: The influence of mosquito resting behaviour and associated microclimate for malaria risk. Malar J 2011, 10:183.

18. Imbahale SS, Mukabana WR, Orindi B, Githeko AK, Takken W: Variation in malaria transmission dynamics in three different sites in Western Kenya. J Trop Med 2012, 912408.

19. Omumbo JA, Lyon B, Waweru SM, Connor SJ, Thomson MC: Raised temperatures over the Kericho tea estates: revisiting the climate in the East African highlands malaria debate. Malar J 2011, 10:12.

20. Kuhn KG, Campbell-Lendrum DH, Davies CR: A continental risk map for malaria mosquito (Diptera: Culicidae) vectors in Europe. J Med Ent 2002, 39:621-630.

21. Paaijmans KP, Read AF, Thomas MB: Understanding the link between malaria risk and climate. Proc Natl Acad Sci USA 2009, 106:13844-13849.

22. Paaijmans KP, Blanford S, Bell AS, Blanford JI, Read AF, Thomas MB: Influence of climate on malaria transmission depends on daily temperature variation. Proc Natl Acad Sci USA 2010, 107:15135-15139.

23. Nagpal BN, Srivastava A, Dash AP, Valecha N: Resting behaviour of Anopheles stephensi type form to assess its amenability to control malaria through indoor residual spray. J Vector Borne Dis 2012, 49:175-180.

24. Batra CP, Reuben R, Das PK: Studies of day-time resting places of Anopheles stephensi Liston in Salem (Tamil Nadu). Indian J Med Research 1979, 69:583-588.

25. Hati AK, Chatterjee KK, Biswas D: Daytime resting habits of Anopheles stephensi in an area of Calcutta. Indian J Malariol 1987, 24:85-87.

26. Hati AK, Chatterjee KK, Biswas D, Mukhopadhyay AK, Saha D: A newly discovered habitat of Anopheles stephensi in present-day Calcutta with evidence of natural malaria infection. Trop Geogr Med 1988, 40:376-377.

27. Oringanje C, Alaribe AAA, Oduola AO, Oduwole OA, Adeogun AO, Meremikwu MM, Awolola TS: Vector abundance and species composition of Anopheles mosquitoes in Calabar, Nigeria. J Vector Borne Dis 2011, 48:171-173.

28. Korgaonkar NS, Kumar A, Yadav RS, Kabadi D, Dash AP: Mosquito biting activity on humans \& detection of Plasmodium falciparum infection in Anopheles stephensi in Goa, India. Indian J Med Res 2012, 135:120-126.

29. National Oceanic and Atmospheric Administration Satellite and Information Service: NNDC Climate Data Online. http://www7.ncdc.noaa.gov/CDO/cdo.

30. Field A (Ed): Discovering Statistics Using SPSS. 3rd edition. London: SAGE Publications; 2009.

31. Briere JF, Pracros P, Le Roux AY, Pierre JS: A novel rate model of temperature dependent development for arthropods. Environ Entomol 1999, 28:22-29.

32. Stratman-Thomas WK: The influence of temperature on Plasmodium vivax. Am J Trop Med 1940, 20:703-715.

33. Knowles R, Basu BC: Laboratory studies on the infectivity of Anopheles stephensi. J Mal Inst India 1943, 5:1-29.

34. Siddons LB: Observations on the influence of atmospheric temperature and humidity on the infectivity of Anopheles culicifacies Giles. J Mal Inst India 1944, 5:375-388.

35. Shute $P G$, Maryon M: A study of human malaria oocysts as an aid to species diagnosis. Trans Roy Soc Trop Med Hyg 1952, 46:275-292.

36. Collins WE, Orihel TC, Peter G, Jeter MH, Gell LS: Some observations on the sporogonic cycle of Plasmodium schwetzi, P. vivax and P. ovale in five species of Anopheles. J Eukaryotic Microbiol 1969, 16:589-596. 
37. Bruce-Chwatt LJ: Essential malariology. London: William Heinemann Medical Books Ltd; 1980.

38. Detinova TS: Age-grouping methods in Diptera of medical importance with special reference to some vectors of malaria. Monogr Ser World Health Organ 1962, 47:13-191.

39. Hu XP, Appel AG: Seasonal variation of critical thermal limits and temperature tolerance in Formosan and eastern subterranean termites (Isoptera: Rhinotermitidae). Environ Entomol 2004, 33:197-205.

40. R Development Core Team: R: A language and environment for statistical computing. Vienna, Austria: R Foundation for Statistical Computing; 2008

41. Goward SN: Thermal behavior of urban landscapes and the urban heat island. Phys Geo 1981, 2:19-33.

42. Oke TR: The energetic basis of the urban heat island. Quart J Roy Meteorol Soc 1982, 108:1-24.

43. Vijayalaxmi J, Sekar SP: Indoor thermal performance of ventilated dwellings using fly screens in the hot-humid climate of Chennai, India. J Green Building 2009, 4:150-157.

44. Minakawa N, Omukunda E, Zhou G, Githeko A, Yan G: Malaria vector productivity in relation to the highland environment in Kenya. Am J Trop Med Hyg 2006, 75:448-453.

45. Kirby MJ, Lindsay SW: Responses of adult mosquitoes of two sibling species, Anopheles arabiensis and A. gambiae s.s. (Diptera: Culicidae), to high temperatures. Bull Entomol Research 2004, 94:441-448.

46. Blanford S, Read AF, Thomas MB: Thermal behaviour of Anopheles stephensi in response to infection with malaria and fungal entomopathogens. Malar J 2009, 8:72.

47. Benoit JB, Lopez-Martinez G, Patrick KR, Phillips ZP, Krause TB, Denlinger DL: Drinking a hot blood meal elicits a protective heat shock response in mosquitoes. Proc Natl Acad Sci 2011, 108:8026-8029.

48. Motard A, Marussig M, Rénia L, Baccam D, Landau I, Mattei D, Targett G, Mazier D: Immunization with the malaria heat shock like protein hsp70-1 enhances transmission to the mosquito. Int Immunol 1995, 7:147-150.

49. Kiszewski A, Mellinger A, Spielman A, Malaney P, Sachs SE, Sachs J: A global index representing the stability of malaria transmission. Am J Trop Med Hyg 2004, 70:486-498.

doi:10.1186/1475-2875-12-84

Cite this article as: Cator et al:: Characterizing microclimate in urban malaria transmission settings: a case study from Chennai, India. Malaria Journal 2013 12:84.

\section{Submit your next manuscript to BioMed Central and take full advantage of:}

- Convenient online submission

- Thorough peer review

- No space constraints or color figure charges

- Immediate publication on acceptance

- Inclusion in PubMed, CAS, Scopus and Google Scholar

- Research which is freely available for redistribution 